\title{
Amelioration of doxorubicin-induced cardiotoxicity by resveratrol
}

\author{
SAMEER E. AL-HARTHI ${ }^{1}$, OHOUD M. ALARABI ${ }^{1}$, WAFAA S. RAMADAN ${ }^{2}$, MOHAMED N. ALAAMA $^{3}$, \\ HUDA M. AL-KREATHY ${ }^{1}$, ZOHEIR A. DAMANHOURI ${ }^{1}$, LATEEF M. KHAN ${ }^{1}$ and ABDEL-MONEIM M. OSMAN ${ }^{1}$
}

\author{
Departments of ${ }^{1}$ Pharmacology and ${ }^{2}$ Anatomy; ${ }^{3}$ Cardiology Unit, Department of Medicine, \\ Faculty of Medicine, King Abdulaziz University, Jeddah 21589, Saudi Arabia
}

Received December 8, 2013; Accepted January 1, 2014

DOI: $10.3892 / \mathrm{mmr} .2014 .2384$

\begin{abstract}
Doxorubicin (DOX), is a highly active anticancer agent, but its clinical use is limited by its severe cardiotoxic side-effects associated with increased oxidative stress and apoptosis. Resveratrol (RSVL) is a naturally occurring polyphenolic compound (trans-3,5,4'-trihydroxystilbene) found primarily in root extracts of the oriental plant Polygonum cuspidatum and of numerous additional plant species. It has recently been shown that RSVL has a number of beneficial effects in different biological systems, which include anti-oxidant, antineoplastic, anticarcinogenic, cardioprotective and antiviral effects. In this study, we examined whether RSVL has protective effects against DOX-induced free radical production and cardiotoxicity in male rats. The tested dose of DOX $(20 \mathrm{mg} / \mathrm{kg})$ caused a significant increase in the serum activities of the cardiac enzymes lactate dehydrogenase (LDH) and creatine phosphokinase (CPK) and the level of malondialdehyde (MDA) in the heart tissue. However, there was a significant decrease in the glutathione level in the heart tissue. Simultaneous treatment of rats with RSVL $[10 \mathrm{mg} / \mathrm{kg}$, intraperitoneal (i.p.) injection] reduced the activity of LDH and CPK and significantly reduced MDA production in the heart. The total antioxidant capacity was increased following RSVL administration. Electron microscopy examination of the heart tissue showed that DOX treatment results in massive fragmentation and lysis of the myofibrils, and that mitochondria show either vacuolization or complete loss of the cristae. Simultaneous treatment with RSVL ameliorated the effect of DOX administration on cardiac tissue, with cardiomyocytes appearing normal compared to the control samples, and mitochondria retaining their normal structure.
\end{abstract}

\section{Introduction}

Doxorubicin (DOX) was introduced in cancer therapy in the late 1960s. It has emerged as one of the most potent

Correspondence to: Professor Abdel-Moneim Mahmoud Osman, Department of Pharmacology, Faculty of Medicine, King Abdulaziz University, Abdullah Sulayman Street, Jeddah 21589, Saudi Arabia

E-mail: moneimosman@hotmail.com

Key words: doxorubicin, resveratrol, cardioprotection broad-spectrum antitumor anthracycline antibiotics. DOX can be administered as a single agent or in combination with other chemotherapeutic agents. It is widely used in the treatment of a variety of cancer types, including leukemia, lymphoma, soft-tissue sarcoma and solid tumors. Its cytotoxic effects on malignant cells, however, are complicated by an increase in the risk of cardiotoxicity $(1,2)$.

It is notable that DOX directly generates free radicals. Moreover, through redox cycling, DOX is a strong chemical catalyst for the production of oxygen radicals $(3,4)$. Furthermore, a reduction in the quantity of endogenous antioxidants has been demonstrated following DOX treatment (5). The oxidative damage induced by DOX affects the lysosomes, microfibrils, mitochondria and the sarcoplasmic reticulum (6-9). Eventually, these intracellular modifications result in increased apoptosis in the cardiac myocytes.

In an attempt to minimize the effective chemotherapeutic dose of DOX and thereby, its side-effects, a variety of approaches have been adopted. One of these approaches is the screening of natural compounds with chemopreventive or anticancer properties that can be used in combination with DOX. Resveratrol (RSVL) is a naturally occurring polyphenolic compound (trans-3,5,4-trihydroxystilbene) found primarily in root extracts of the oriental plant Polygonum cuspidatum and of numerous plant species (10). It is highly abundant in the skin of red grapes and moderately abundant in peanuts and blueberries (10).

A previous study by our group using a model of DOX-induced heart damage in rats showed that pretreatment with aged garlic extract, a strong antioxidant, provides protection from DOX-induced myocardial cell damage (11). Therefore, the present study was undertaken to test whether RSVL, a compound with known antioxidant properties, can also protect cells from DOX-induced cardiotoxicity. Using electron microscopy, we studied the subcellular effects of DOX in the heart and the underlying mechanisms of these effects.

\section{Materials and methods}

Reagents. DOX hydrochloride and RSVL were purchased from Sigma-Aldrich (St. Louis, MO, USA). The stock solution of both drugs was dissolved in normal saline and preserved at $-20^{\circ} \mathrm{C}$. The solutions were diluted in normal saline to reach the desired final concentration immediately prior to each experiment. 


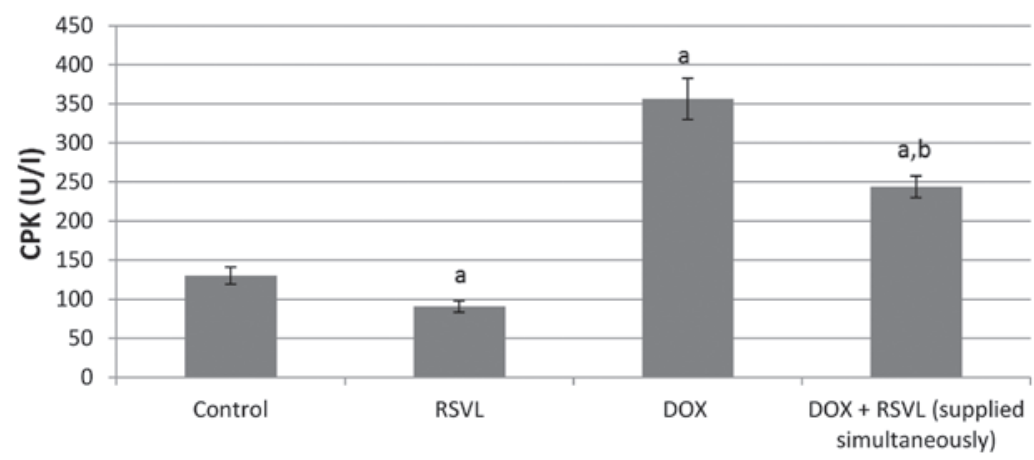

Figure 1. Effect of doxorubicin (DOX) and/or resveratrol (RSVL) on the level of serum creatine phosphokinase (CPK). Bars represent standard error of the mean from measurements in six rats. ${ }^{a}$ Significantly different from control (normal saline treatment) at $\mathrm{P}<0.05$; ${ }^{\mathrm{b}}$ significantly different from $\mathrm{DOX}$ treatment at $\mathrm{P}<0.05$.

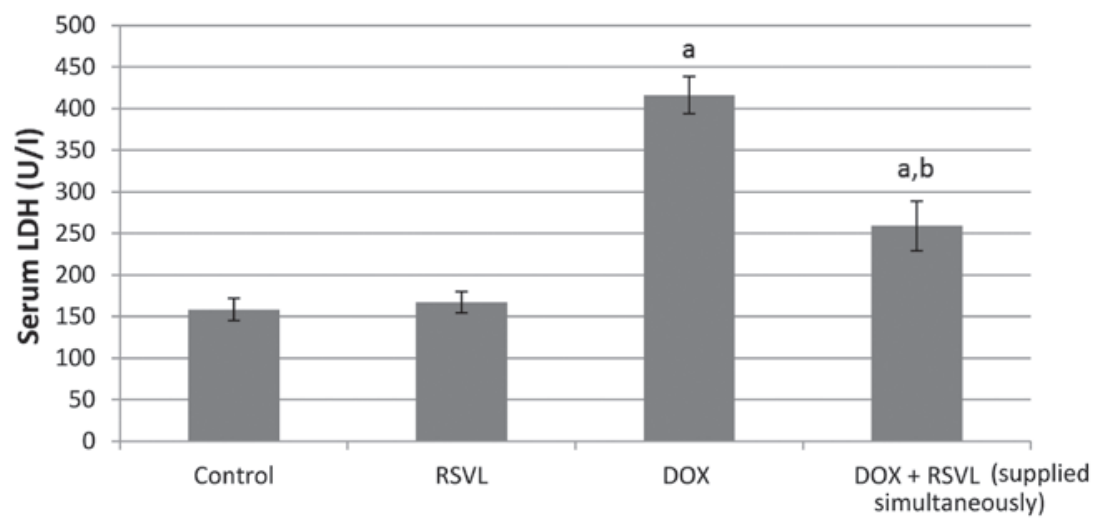

Figure 2. Effect of doxorubicin (DOX) and/or resveratrol (RSVL) on the level of serum lactate dehydrogenase (LDH). Bars denote standard error of the mean from measurements in six rats. ${ }^{a}$ Significantly different from control (normal saline treatment) at $\mathrm{P}<0.05 ;{ }^{\mathrm{b}}$ significantly different from $\mathrm{DOX}$ treatment at $\mathrm{P}<0.05$.

Animals. Male Wistar albino rats (8-10 weeks of age, $180-200 \mathrm{~g}$ body weight) were obtained from the King Fahd Medical Research Center, King Abdulaziz University, Jeddah, Saudi Arabia. The animals were acclimatized for one week. Rats had access to a commercial balanced diet and water ad libitum throughout the experiment. This study was approved by the Ethical Committee of the King Abdulaziz Hospital.

Experimental protocol. A total of 24 male Wistar rats were divided into 4 equal groups consisting of 6 animals each and housed in a room with regular 12-h light/dark cycle with free access to food and water. Two groups (I and II) were used as controls, and rats in these groups received an intraperitoneal (i.p.) injection of normal saline $(0.9 \%)$ and RSVL $(10 \mathrm{mg} / \mathrm{kg}$, i.p.), respectively. Group III received DOX (20 mg/kg), while Group IV received DOX (20 mg/kg) simultaneously with RSVL $(10 \mathrm{mg} / \mathrm{kg})$. At the end of the experiment, i.e. $48 \mathrm{~h}$ after the DOX injection, rats were anesthetized and blood samples were collected from the ophthalmic artery in the orbital rim prior to sacrifice. The serum was isolated from these samples and the heart specimens were fixed in $2.5 \%$ formaldehyde and $2.5 \%$ glutaraldehyde for electron microscopy.

Assessment of cardiac enzyme activities. Plasma total lactate dehydrogenase (LDH) and total creatine phosphokinase (CPK) activities were determined using commercial kits from Randox Laboratories (Crumlin, UK) and Spinreact (Girona, Spain), respectively.
Determination of lipid peroxides. Frozen heart samples were thawed, rinsed successively with $0.9 \% \mathrm{NaCl}$ and with cold $\left(4^{\circ} \mathrm{C}\right) 20 \mathrm{mM}$ Tris- $\mathrm{HCl}$, followed by homogenization in a Branson sonifier (250; VWR International, Danbury, CT, USA). The homogenates were diluted with cold $20 \mathrm{mM}$ Tris- $\mathrm{HCl}$ and centrifuged $\left(10 \mathrm{~min}\right.$ at $\left.4{ }^{\circ} \mathrm{C} ; 3,000 \mathrm{x} \mathrm{g}\right)$. The levels of malondialdehyde (MDA) and reduced glutathione were assayed spectrophotometrically at $534 \mathrm{~nm}$ using commercial kits from Randox Laboratories in accordance with the instructions of the manufacturer.

Determination of total antioxidant capacity (TAC) in the serum. TAC was determined using a previously described method (12) based on the quenching of the radical ABRS ${ }^{+}$ (2,2-azino-di(3-ethyl benzthiazolin sulphonate cation) by antioxidants. The Total Antioxidant Assay kit (NX2332; Randox Laboratories) was used for this purpose.

Examination of heart sections by electron microscopy. Preparation of samples for electron microscopy was performed as follows: biopsies were placed into fixative buffer containing $2.5 \%$ formaldehyde and $2.5 \%$ glutaraldehyde for $\geq 1 \mathrm{~h}$. Tissue samples were rinsed three times for 15 min each with $0.075 \mathrm{M}$ sodium phosphate buffer, after which the samples were placed in a $1 \%$ osmium tetraoxide $\left(\mathrm{OsO}_{4}\right)$ secondary fixative buffer for $1 \mathrm{~h}$. Samples were finally embedded with Quetol epoxy resin (Polysciences Europe GmbH, Eppelheim, Germany) in rubber moulds and allowed to polymerize in an oven at $60^{\circ} \mathrm{C}$ 


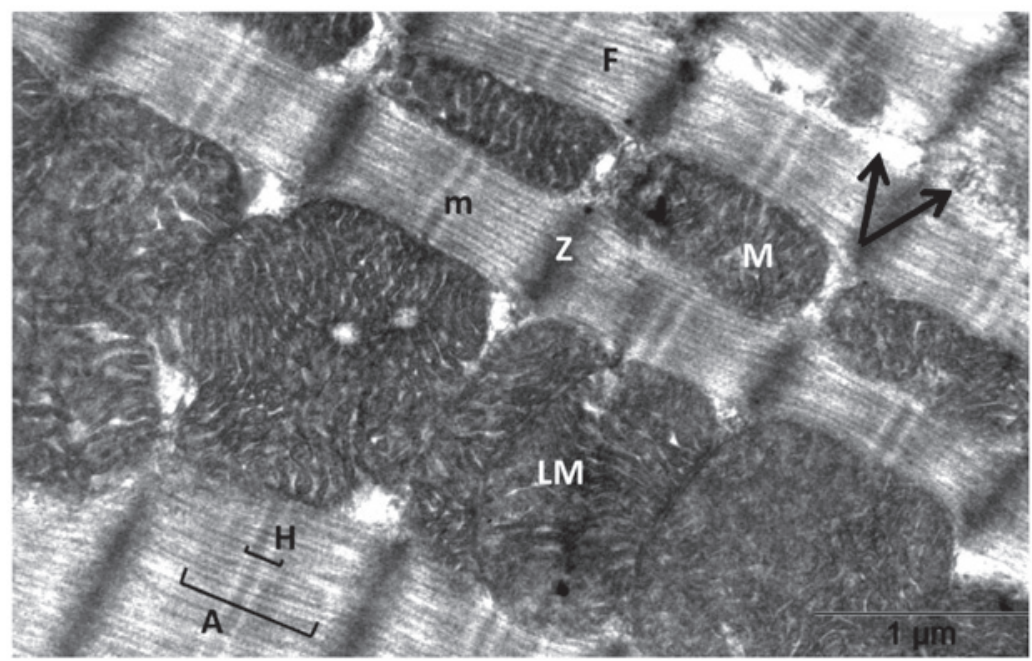

Figure 3. Electron micrograph of the left ventricular myocardium of a control rat showing regular arrangement of the myofibrils (F) and the mitochondria (M), with abundant regular cristae in between. A large mitochondrion (LM) is also shown. Tubular and vesicular segments of the sarcoplasmic reticulum are interspersed between the myofibrils (black arrows). Striations are evident among the well-organized myofilaments with lines (m) intersecting the $\mathrm{H}$ bands. The $\mathrm{A}$ band and $\mathrm{Z}$ lines are also observed.

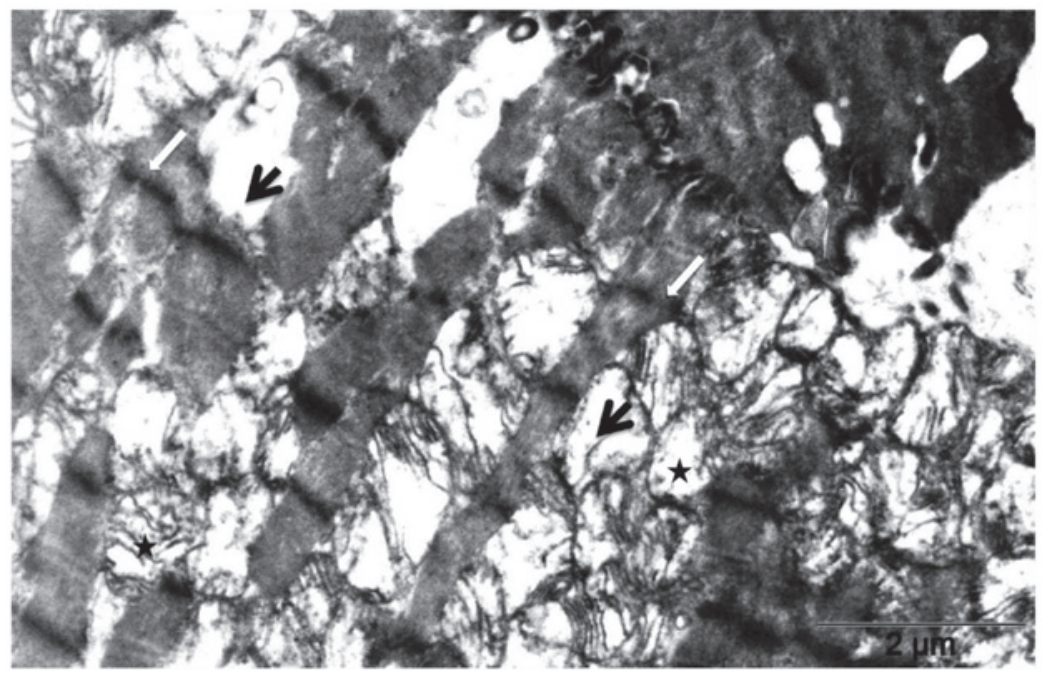

Figure 4. Electron micrograph of the left ventricular myocardium of a rat intraperitoneally injected with $20 \mathrm{mg} / \mathrm{kg}$ doxorubicin shows massive fragmentation and lysis of the myofibrils (black arrows). Mitochondria ( $\star$ ) show either vacuolization or complete loss of the cristae. Interruption of $\mathrm{Z}$ lines (white arrows) is also observed.

for $\sim 39$ h prior to ultramicrotomy. Samples were subsequently cut at a 70-100 nm thickness, placed onto copper grids and stained. Transmission electron microscopy analysis was then performed using a JEM $2100 \mathrm{~F}$ transmission electron microscope (JEOL, Peabody, MA, USA).

Statistical analysis. Results were expressed as the mean \pm standard error of the mean. Comparisons between different groups were carried out by one way analysis of variance tests. $P \leq 0.05$ was considered to indicate a statistically significant difference.

\section{Results}

Effect of RSVL on DOX-induced cardiotoxicity. Treatment of rats with DOX $(20 \mathrm{mg} / \mathrm{kg}$, i.p.) caused a significant 2.7-fold increase in the activity of both serum CPK and LDH enzymes (Figs. 1 and 2). Simultaneous treatment with DOX and RSVL reduced the effect of DOX by 1.9- and 1.6-fold, respectively.

The comparison of heart tissues of control rats (Fig. 3) and of rats treated with $20 \mathrm{mg} / \mathrm{kg}$ DOX (Fig. 4) by electron microscopy revealed massive fragmentation and lysis of the myofibrils upon DOX treatment (Fig. 4, black arrows). Mitochondria showed either vacuolization or complete loss of the cristae. Interruption of $\mathrm{Z}$ lines (Fig. 4, white arrows) was also evident. The heart tissues showed organized myofibrils with mitochondria in between upon simultaneous treatment with DOX with RSVL ((Fig. 5). The mitochondria retained a normal structure similarly to those of control rats (Fig. 3). Focal areas of myofibrilar loss (Fig. 5, black arrow) and dilated sarcoplasmic reticulum (Fig. 5, grey dashed arrow) were observed. Rats treated with RSVL alone (Fig. 6) showed a generally organized myofibril architecture. The mitochondria in these rats showed a regular cristae pattern. 


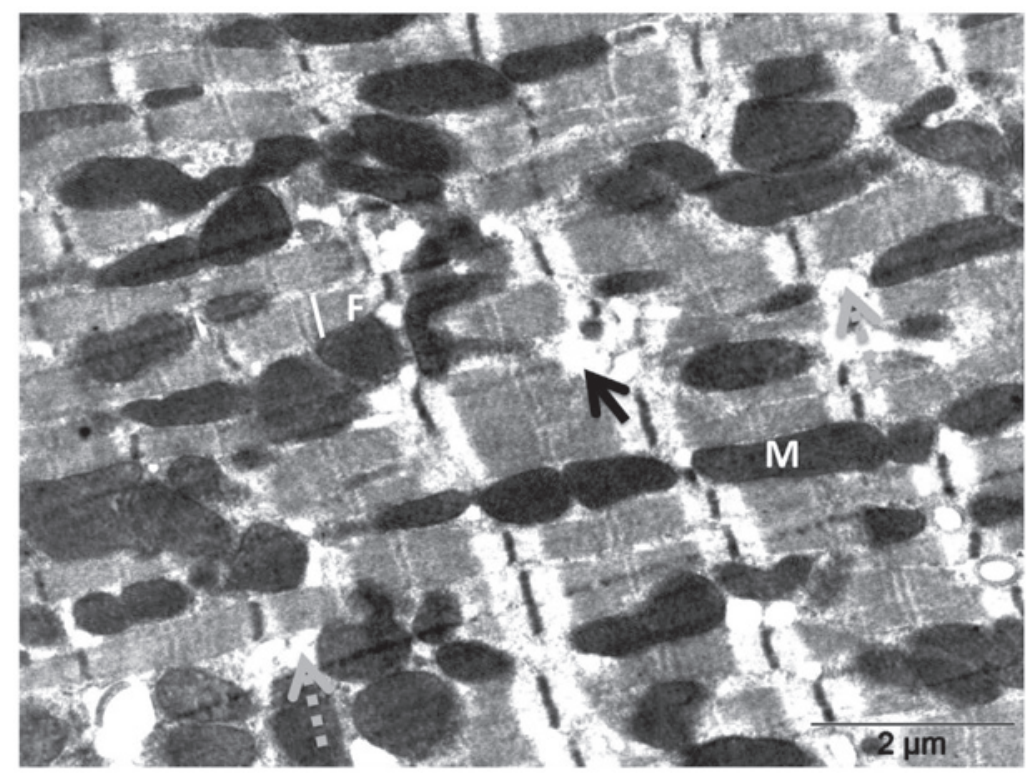

Figure 5. Electron micrograph of left ventricular myocardium of a rat treated with doxorubicin [(20 mg/kg, intraperitoneal (i.p.) injection] simultaneously with resveratrol $(10 \mathrm{mg} / \mathrm{kg}$, i.p.) showing organized myofibrils (F) with mitochondria $(\mathrm{M})$ in between. The mitochondria retain a normal structure. Focal areas of myofibrilar loss (black arrow) and dilated sarcoplasmic reticulum (grey dashed arrow) are observed.

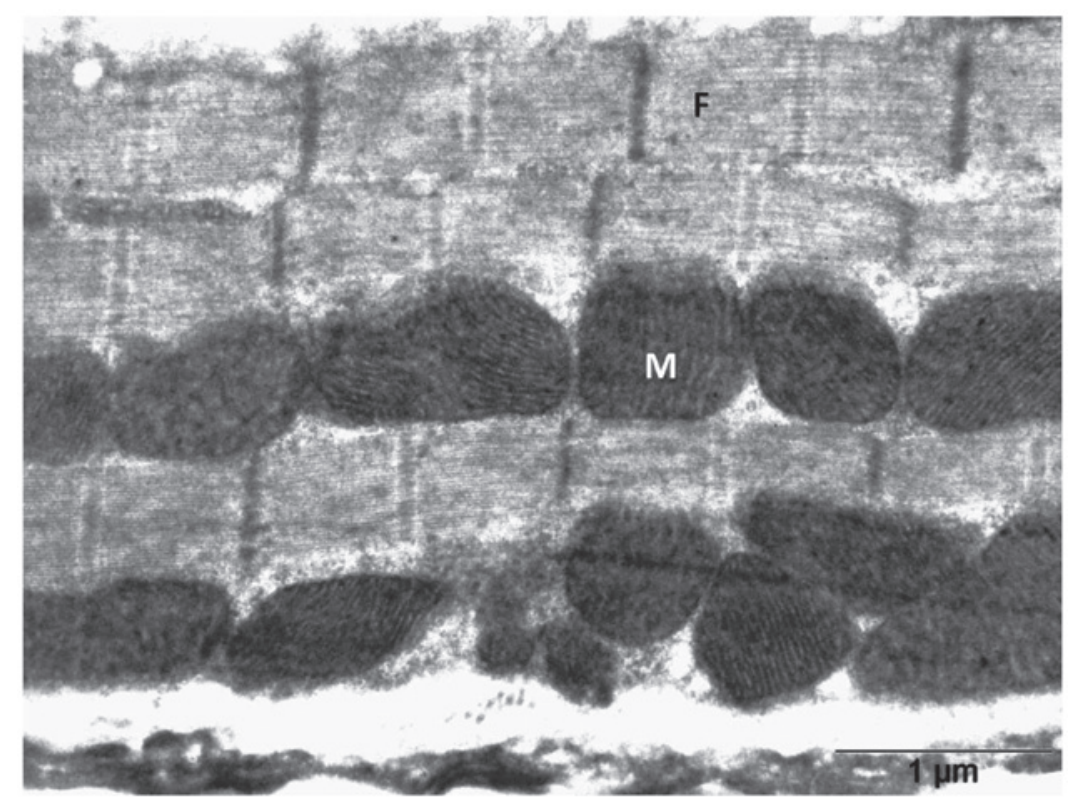

Figure 6. Electron micrograph of the left ventricular myocardium of a rat treated with $10 \mathrm{mg} / \mathrm{kg}$, intraperitoneal resveratrol showing a generally organized myofibril (F) architecture. The mitochondria $(\mathrm{M})$ show regular cristae patterns.

Effect of RSVL on DOX-induced changes in the levels of MDA and reduced glutathione. Table I and Fig. 7 show the effect of combined RSVL and DOX treatment on the levels of MDA and reduced glutathione in the heart tissues. DOX treatment caused a $12 \%$ increase and a $21 \%$ decrease in the MDA and reduced glutathione level, respectively.

Evaluation of serum TAC. Fig. 8 shows the effect of DOX $(20 \mathrm{mg} / \mathrm{kg}$, i.p.) and/or RSVL (10 mg/kg, i.p.) on the TAC, meaured in the serum of treated rats. An important and significant decrease $(32 \%)$ in TAC was observed in DOX-treated rats compared to controls, while combined treatment with RSVL and DOX did not significantly affect TAC compared to control treatment.

\section{Discussion}

Anthracyclines are used to treat a variety of cancer types, but are widely associated with irreversible cardiomyopathy. The mechanism of DOX-induced oxidative stress is the formation of an anthracycline-iron $\left(\mathrm{Fe}^{2+}\right)$ free radical complex. The latter reacts with hydrogen peroxide to produce the hydroxyl $\left(\mathrm{OH}^{*}\right)$ radical (13). Iron chelators and free radical scavengers may provide cardiac protection by preventing the formation of the 


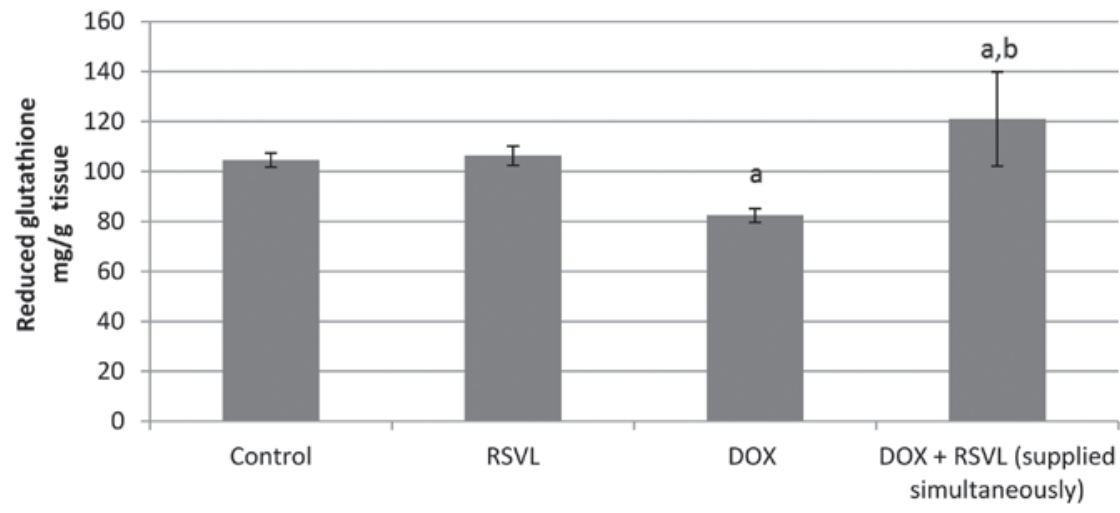

Figure 7. Effect of doxorubicin (DOX) and/or resveratrol (RSVL) on the level of reduced glutathione in the heart homogenate of rats. Bars denote standard

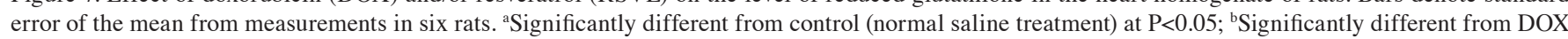
treatment at $\mathrm{P}<0.05$.

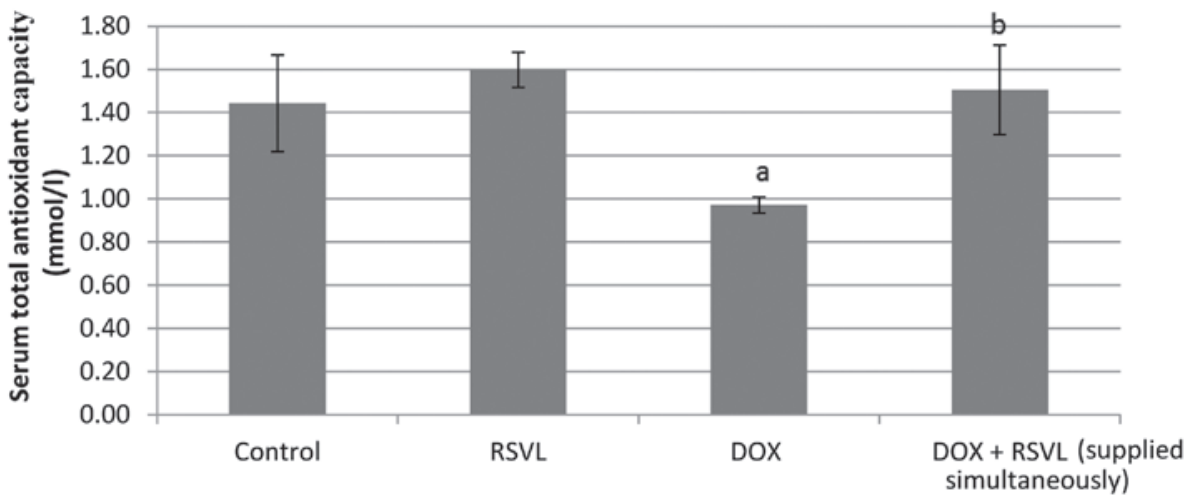

Figure 8. Effect of doxorubicin (DOX) and/or resveratrol (RSVL) on the total serum antioxidant activity in rats. Bars denote standard error of the mean from measurements in six rats. ${ }^{a}$ Significantly different from control (normal saline treatment) at $\mathrm{P}<0.05$; ${ }^{\mathrm{b}}$ significantly different from $\mathrm{DOX}$ treatment at $\mathrm{P}<0.05$.

Table I. Effect of doxorubicin (DOX) and/or resveratrol (RSVL) on the malondialdehyde (MDA) level in the heart homogenate of rats.

\begin{tabular}{lc} 
Treatment & MDA level \\
\hline Control (normal saline) & $1,164.10 \pm 31.81$ \\
RSVL & $1,108.29 \pm 44.60$ \\
DOX & $1,345.84^{\mathrm{a}} \pm 62.26$ \\
DOX + RSVL (simultaneously applied) & $1,096.25^{\mathrm{b}} \pm 69.44$
\end{tabular}

Data are presented as mean $(\mathrm{nmol} / \mathrm{g}$ tissue $) \pm$ standard error of the

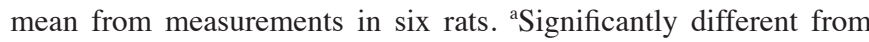
control treatment at $\mathrm{P}<0.05$; ${ }^{\mathrm{b}}$ significantly different from $\mathrm{DOX}$ treatment at $\mathrm{P}<0.05$. Control, DOX and RSVL treatments were performed with intraperitoneal injection of normal saline (0.9\%), DOX (20 mg/kg) and RSVL (10 mg/kg), respectively.

reactive hydroxyl radical and by scavenging radicals that have been formed. The iron chelator ICRF-187 has been shown to protect against DOX-induced cardiotoxicity. However, its clinical success is limited because it increases hematotoxicity in cancer patients $(14,15)$. This study investigated the potential cardioprotective effects of RSVL against DOX-induced cardiotoxicity. In animal models of cardiovascular disease,
RSVL has been shown to protect the heart from ischemia reperfusion injury, reduce blood pressure and cardiac hypertrophy on hypertensive animals, and inhibit the progression of atherosclerosis (16). The exact mechanism underlying the cardioprotective effect of RSVL is not known, but previous studies reporting that RSVL can inhibit the DOX-induced rapid increase in reactive oxygen species (ROS) in the mitochondria of cardiac cells (17) by increasing superoxide dismutase activity (18), suggest that the antioxidant properties of RSVL may play a role in its cardioprotective effects. In this context, the present study was designed to investigate the effect of RSVL treatment on oxidative stress and examine the subcellular effect of DOX in the heart, along with the underlying mechanisms. The tested dose of DOX induced marked and acute cardiotoxicity in rats, manifested by an increase in the plasma CPK and LDH activities and confirmed by electron microscopy, which revealed changes in the heart tissue, such as massive fragmentation and lysis of the myofibrils and vacuolization or complete loss of the cristae in the mitochondria. It is well known that certain enzymes (CPK, LDH) are released from the heart muscle cells when it is injured, and the magnitude of CPK and LDH activities in the blood following myocardial injury reflects the extent of damage in its musculature (19).

The mechanism of DOX-induced cardiotoxicity has been reported to involve formation of superoxide anions 
and their derivatives, particularly highly reactive and damaging hydroxyl radicals, which cause peroxidation of the cell membrane lipids $(20,21)$. Our results are in agreement with other studies that reported cardiac toxicity following DOX treatment (22-25). The mechanism of DOX-induced cardiotoxicity has been investigated by numerous research groups. In terms of specific organ toxicity, lipid peroxidation has been highlighted as the primary mechanism underlying DOX-induced cardiac toxicity $(7,26)$. A significant increase in lipid peroxidation was also observed in our study, as manifested by the increased plasma MDA level in the DOX-treated group (Table I).

The increase in CK activity following DOX treatment was prevented by simultaneous treatment with RSVL. In line with the hypothesis that RSVL acts through inhibition of the DOX-induced rapid accumulation of ROS in the mitochondria of cardiac cells, we found a significantly reduced level of MDA in cardiac tissue (Table I), normal mitochondrial structures (Fig. 5), and increased TAC (Fig. 8) in DOX + RSVL-treated rats, suggesting that the antioxidant properties of RSVL may play a role in its cardioprotective effects. However, antioxidant therapies have failed to provide satisfactory results in clinical trials (27), casting doubt on the notion that the inhibition of oxidative stress is the sole mechanism underlying the cardioprotective effects of RSVL. Recently, Osman et al (25) showed that RSVL increases the DOX uptake into Ehrlich ascite cells, allowing to use a reduced dose of DOX with reduced side-effects. In conclusion, RSVL can protect cardiac cells from the deleterious effects of DOX via its antioxidant properties. Additional clinical trials with RSVL may allow to further elucidate its protective role against agents that induce tissue-damaging effects, while further studies are necessary to reveal the molecular basis of such effects.

\section{References}

1. Lefrak EA, Pitha J, Rosenheim S and Gofottiebm JA: A clinicopathological analysis of adriamycin cardiotoxicity. Cancer 32: 302-314, 1973.

2. Singal PK and Iliskovic N: Doxorubicin-induced cardiomyopathy. N Engl J Med 339: 900-905, 1998.

3. Doroshow JH: Effect of anthracycline antibiotics on oxygen radical formation in rat heart. Cancer Res 43: 460-472, 1983.

4. Powis G: Free radical formation by antitumor quinones. Free Radic Biol Med 6: 63-101, 1989.

5. Olson RD and Mushlin PS: Doxorubicin cardiotoxicity: analysis of prevailing hypotheses. FASEB J 4: 3076-3086, 1990.

6. Ogura R, Sugiyama M, Haramaki N and Hidaka T: Electron spin resonance studies on the mechanism of adriamycin-induced heart mitochondrial damages. Cancer Res 51: 3555-3558, 1991

7. Myers CE, McGuire WP, Liss RH, Ifrim I, Grotzinger K and Young RC: Adriamycin: the role of lipid peroxidation in cardiac toxicity and tumor response. Science 197: 165-167, 1977.
8. Mimnaugh EG, Trush MA, Bhatnagar M and Gram TE: Enhancement of reactive oxygen-dependent mitochondrial membrane lipid peroxidation by the anticancer drug adriamycin. Biochem Pharmacol 34: 847-856, 1985.

9. Singal PK, Deally CM and Weinberg LE: Subcellular effects of adriamycin in the heart: a concise review. J Mol Cell Cardiol 19: 817-828, 1987.

10. Goswami SK and Das DK: Resveratrol and chemoprevention. Cancer Lett 284: 1-6, 2009.

11. Alkreathy H, Damanhouri ZA, Ahmed N, Slevin M, Ali SS and Osman AM: Aged garlic extract protects against doxorubicin-induced cardiotoxicity in rats. Food Chem Toxicol 48: 951-956, 2010.

12. Miller NJ, Rice-Evans C, Davies MJ, Gopinathan V and Milner A: A novel method for measuring antioxidant capacity and its application to monitoring the antioxidant status in premature neonates. Clin Sci (Lond) 84: 407-412, 1993.

13. Sugioka K and Nakano M: Mechanism of phospholipid peroxidation induced by ferric ion-ADP-adriamycin-co-ordination complex. Biochim Biophys Acta 713: 333-343, 1982.

14. Sparano JA: Use of dexrazoxane and other strategies to prevent cardiomyopathy associated with doxorubicin-taxane combinations. Semin Oncol 25 (Suppl 10): 66-71, 1998.

15. Speyer JL, Green MD, Zeleniuch-Jacquotte A, Wernz JC, Rey M, Sanger J, Kramer E, Ferrans V, Hochster H, Meyers M, et al: ICRF-187 permits longer treatment with doxorubicin in women with breast cancer. J Clin Oncol 10: 117-127, 1992.

16. Li H, Xia N and Förstermann U: Cardiovascular effects and molecular targets of resveratrol. Nitric Oxide 26: 102-110, 2012.

17. Danz ED, Skramsted J, Henry N, Bennett JA and Keller RS: Resveratrol prevents doxorubicin cardiotoxicity through mitochondrial stabilization and the Sirtl pathway. Free Radic Biol Med 46: 1589-1597, 2009.

18. Tatlidede E, Sehirli O, Velioğlu-Oğünc A, Cetinel S, Yeğen BC, Yarat A, Süleymanoğlu S and Sener G: Resveratrol treatment protects against doxorubicin-induced cardiotoxicity by alleviating oxidative damage. Free Radic Res 43: 195-205, 2009.

19. Preus M, Bhargava AS, Khater AE and Günzel P: Diagnostic value of serum creatine kinase and lactate dehydrogenase isoenzyme determinations for monitoring early cardiac damage in rats. Toxicol Lett 42: 225-233, 1988.

20. Osman AM, Al-Shabanah OA and Al-Harbi MM: Effect of desferrioxamine on doxorubicin-induced cardiotoxicity and haematotoxicity in mice. Med Sci Res 21: 193-194, 1993.

21. Hemnani T and Parihar MS: Reactive oxygen species and oxidative DNA damage. Indian J Physiol Pharmacol 42: 440-452, 1998.

22. Van Vleet JF, Ferrans VJ and Weirich WE: Cardiac disease induced by chronic adriamycin administration in dogs and an evaluation of vitamin $\mathrm{E}$ and selenium as cardioprotectants. Am J Pathol 99: 13-42, 1980.

23. Nagi MN and Mansour MA: Protective effect of thymoquinone against doxorubicin-induced cardiotoxicity in rats: a possible mechanism of protection. Pharmacol Res 41: 283-289, 2000.

24. Al-Majed AA, Gado AM, Al-Shabanah OA and Mansour MA: Alpha-lipoic acid ameliorates myocardial toxicity induced by doxorubicin. Pharmacol Res 46: 499-503, 2002.

25. Osman AM, Al-Harthi SE, Alarabi OM, Elshal MF, Ramadan WS, Alaama MN, Al-Kreathy HM, Damanhouri ZA and Osman OH: Chemosensetizing and cardioprotective effects of resveratrol in doxorubicin-treated animals. Cancer Cell Int 13: 52, 2013.

26. Singal PK and Pierce GN: Adriamycin stimulates low-affinity $\mathrm{Ca}^{2+}$ binding and lipid peroxidation but depresses myocardial function. Am J Physiol 250: H419-H425, 1986.

27. Gianni L, Herman EH, Lipshultz SE, Minotti G, Sarvazyan N and Sawyer DB: Anthracycline cardiotoxicity: from bench to bedside. J Clin Oncol 26: 3777-3784, 2008. 